\title{
Regulation of arsenite oxidation by the phosphate two-component system PhoBR in Halomonas sp. HAL1
}

\author{
Fang Chen, Yajing Cao, Sha Wei, Yanzhi Li, Xiangyang Li, Qian Wang * and Gejiao Wang * \\ State Key Laboratory of Agricultural Microbiology, Huazhong Agricultural University, Wuhan, China
}

Previously, the expression of arsenite [As(III)] oxidase genes aioBA was reported to be regulated by a three-component regulatory system, AioXSR, in a number of As(III)-oxidizing bacterial strains. However, the regulation mechanism is still unknown when aioXSR genes are absent in some As(III)-oxidizing bacterial genomes, such as in Halomonas sp. HAL1. In this study, transposon mutagenesis and gene knock-out mutation were performed, and two mutants, HAL1-phoR ${ }_{931}$ and HAL1- $\triangle$ phoB, were obtained in strain HAL1. The phoR and phoB constitute a two-component system which is responsible for phosphate $(\mathrm{Pi})$ acquisition and assimilation. Both of the mutants showed negative As(III)-oxidation phenotypes in low Pi condition $(0.1 \mathrm{mM})$ but not under normal Pi condition (1 mM). The phoBR complementation strain HAL1- $\triangle$ phoB-C reversed the mutants' null phenotypes back to wild type status. Meanwhile, lac $Z$ reporter fusions using pCM-lacZ showed that the expression of phoBR and aioBA were both induced by As(III) but were not induced in HAL1-phoRg31 and HAL1- $\triangle$ phoB. Using 15 consensus Pho box sequences, a putative Pho box was found in the aioBA regulation region. PhoB was able to bind to the putative Pho box in vivo (bacterial one-hybrid detection) and in vitro (electrophoretic mobility gel shift assay), and an 18-bp binding sequence containing nine conserved bases were determined. This study provided the evidence that PhoBR regulates the expression of aioBA in Halomonas sp. HAL1 under low Pi condition. The new regulation model further implies the close metabolic connection between $\mathrm{As}$ and $\mathrm{Pi}$.

This article was submitt Microbiological Chemistry and Geomicrobiology,

a section of the journal

Frontiers in Microbiology

Received: 22 June 2015

Accepted: 21 August 2015

Published: 09 September 2015

Citation:

Chen F, Cao Y, Wei S, Li Y, Li X,

Wang $Q$ and Wang $G$ (2015)

Regulation of arsenite oxidation by the phosphate two-component system PhoBR in Halomonas sp. HAL 1 .

Front. Microbiol. 6:923. doi: 10.3389/fmicb.2015.00923

Keywords: arsenite oxidation, arsenite oxidase AioBA, phosphate two-component system, regulation of gene expression, Halomonas sp. HAL1

\section{Introduction}

Arsenic (As) is a toxic metalloid that is widely distributed in the environment and primarily exists in the inorganic forms of arsenite [As(III)] and arsenate [As(V)] (Kulp et al., 2004; Sharma and Sohn, 2009). Bacterial As(III) oxidation is an elaborate regulation process because it is not only a microbial detoxification mechanism but, in some cases, also an exergonic process that can support bacterial growth (Santini et al., 2000; Rhine et al., 2007; Kulp et al., 2008; Wang et al., 2015). The As(III) oxidase AioBA is responsible for most bacterial As(III) oxidation, and its expression was reported to be controlled by the two-component system AioSR via phosphorylation (Kashyap et al., 2006; Sardiwal et al., 2010). This regulatory model was expanded to the three-component system 
AioXSR in Agrobacterium tumefaciens 5A (Liu et al., 2012). The periplasmic As(III) binding protein AioX changes conformation after binding with $\mathrm{As}$ (III) and interacts with AioS, therefore involved in the regulation of As(III) oxidation with AioSR (Liu et al., 2012).

As opposed to A. tumefaciens $5 \mathrm{~A}$, in which aioXSR and aioBA are in the same operon and are transcribed in the same direction (Kang et al., 2012b; Liu et al., 2012), the aioXSR and aioBA are in different operons and are divergently transcribed in Thiomonas arsenitoxydans 3As (Moinier et al., 2014). The metalloprotein AioF belonging to the ArsR/SmtB family was found to be able to bind specifically to the regulatory region of the aio operon at two distinct positions (Moinier et al., 2014). It involved in the tightly control of the aioBA expression functioning together with AioXSR in T. arsenitoxydans 3As (Moinier et al., 2014). However, the three-component system AioXSR is absent in the genomes of some As(III)-oxidizing bacteria (e.g., Halomonas sp. HAL1) (Lin et al., 2012; Li et al., 2013). In these cases, the regulation mechanism responsible for the expression of aioBA remains unknown.

Two-component system PhoBR was reported to be responsible for the regulation of phosphate uptake and assimilation in Escherichia coli and other species (Wanner, 1996; Hsieh and Wanner, 2010). It is composed of a transmembrane sensory histidine kinase (HK) PhoR, and a response regulator (RR) PhoB (Hsieh and Wanner, 2010). Under phosphatedeficient conditions, PhoR catalyzes autophosphorylation on the conserved His residue, and the His-bound phosphoryl moiety is subsequently transferred to an Asp residue of PhoB. Then, the phosphorylated PhoB dimers and binds to a specific DNA sequence termed as Pho box. The DNA sequence is formed by two 7-bp direct repeats separated by a conserved 4-bp AT-rich spacer. Upon binding to the DNA, PhoB recruits the RNA polymerase and interacts with the RNA polymerase $\sigma^{70}$ subunit to control the transcription of downstream genes (Makino et al., 1993; Hsieh and Wanner, 2010; Blanco et al., 2011, 2012). In E. coli, 31 genes in nine transcripts (eda, phnCDEFGHIJKLMNOP, phoA, phoBR, phoE, phoH, psiE, pstSCAB-phoU, and ugpBAECQ) were directly controlled by PhoBR (Hsieh and Wanner, 2010). In addition, PhoBR was reported to regulate the expression of genes related to chemotaxis, antibiotic resistance, and virulence attenuation (Pratt et al., 2009; Crepin et al., 2011; Srinivasan et al., 2012), indicating that PhoBR plays a key role in a variety of bacterial physiological metabolisms. Previously, it was reported that the phosphate $(\mathrm{Pi})$ stress-response genes phoB, pstS, and phoU are intricately co-regulated with As(III) oxidation genes in A. tumefaciens 5A (Kang et al., 2012b), indicating PhoBR may involve in the regulation of As(III) oxidation.

In this study, we developed Halomonas sp. HAL1 as a model for understanding the regulation of As(III) oxidation in which AioXSR is absent. Using transposon mutagenesis, gene knock-out mutation and complementation, we found that $p h o R$ and $p h o B$ both influenced As(III) oxidation. Because the Pi concentration is reported to be associated with the expression of the As(III) oxidase AioBA (Kang et al., 2012b), both low and normal Pi conditions are used to conduct the regulation analysis in this study. Bacterial one-hybrid system and electrophoretic

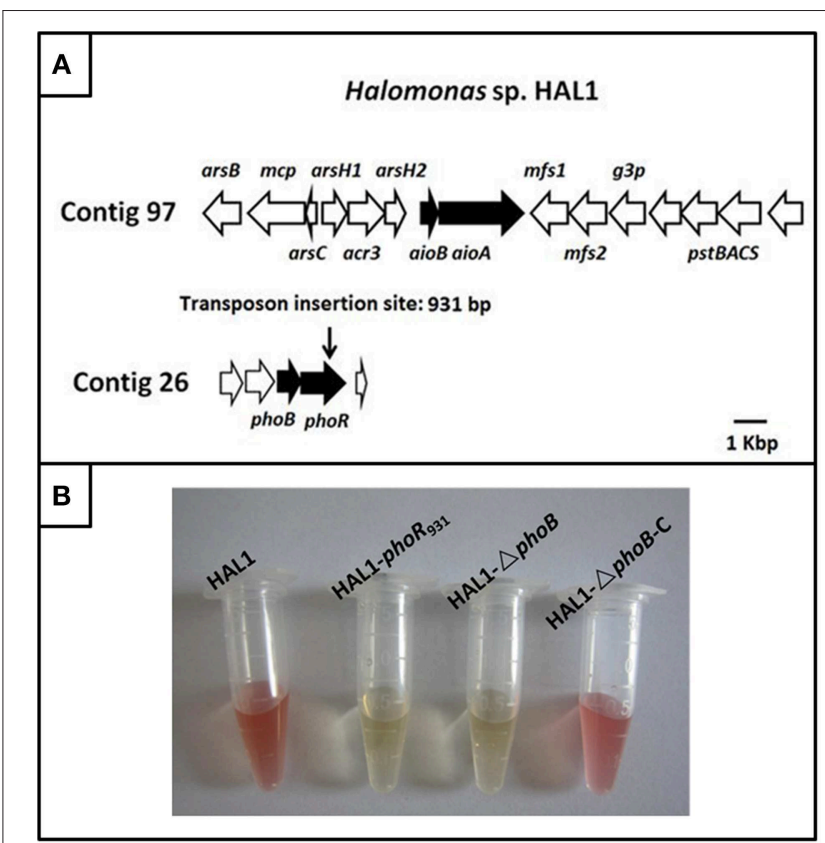

FIGURE 1 | The gene island of As(III) oxidation in Halomonas sp. HAL1 and As(III) oxidation analysis. (A) The gene island of As(III) oxidation. The transposon insertion site of phoR mutant is shown by vertical arrow. (B). As(III) oxidation phenotype of strains HAL1, HAL1-phoR ${ }_{931}$, HAL1- $\Delta$ phoB, and HAL1- $\triangle$ phoB-C. The strains were inoculated into $\mathrm{MMNH}_{4}$ medium containing $0.1 \mathrm{mM} \mathrm{Pi}, 0.8 \mathrm{M} \mathrm{NaCl}$, and $1 \mathrm{mM} \mathrm{As}(\mathrm{III})$. After $7 \mathrm{~d}$ cultivation, the As(III) oxidation was monitored by qualitative $\mathrm{KMnO}_{4}$ biochemical analysis.

mobility shift assay (EMSA) were employed to demonstrate that PhoB could bind with the putative Pho box in the regulatory region of aioBA. The results of this study indicated that the twocomponent system PhoBR can regulate the As(III) oxidation in Halomonas sp. HAL1 under the low Pi condition. The results of this study revealed a novel regulation mechanism of bacterial As(III) oxidation, supplemented the co-regulation mechanism between As and Pi.

\section{Results}

\section{The phoBR System Effected on As(III) Oxidation}

The draft genome sequencing of Halomonas sp. HAL1 revealed that an arsenic gene island located in contig 97 contains the As(III) oxidase genes aioBA, the arsenic efflux genes ars $B$ mcp-arsC-arsH1-acr3-arsH2 and the phosphate-related genes $p s t S C A B$ (Figure 1A) (Lin et al., 2012). However, the threecomponent regulator genes aioXSR were absent in the entire genome (Lin et al., 2012; Li et al., 2013). Genomic analysis revealed that only one copy of phoBR genes locating in contig 26 . The two genes encode two-component system proteins, which is mainly a response to phosphate stress (Lin et al., 2012; Li et al., 2013).

To identify the putative genes related to As(III) oxidation, we used transposon mutagenesis in combination with qualitative $\mathrm{KMnO}_{4}$ screening to isolate the As(III) oxidation mutants. Five mutants disabled for As(III) oxidation were generated by 
screening approximately 2000 tranformates; these five mutants were interrupted in the genes encoding molybdenum cofactors (two mutants), cytochrome c, As(III) oxidase, and PhoR. All of the proteins were reported to be correlated with bacterial As(III) oxidation (Rosen, 1995; Silver and Phung, 2005; Kang et al., 2012b). We paid particular attention to PhoR because we were more interested in the regulation mechanisms of As(III) oxidation. Due to PhoB is the response regulator in the twocomponent system PhoBR, the mutant HAL1- $\triangle p h o B$ and the complementary strain HAL1- $\triangle p h o B-C$ were then constructed using the allelic exchange vector pCM184 and vector pCT-zori (broad-host-range, lac $Z \alpha$ selection marker) constructed in this study (Figure S1). The qualitative $\mathrm{KMnO}_{4}$ tests indicated that the disruption of $p h o R$ or $p h o B$ deprived the As(III) oxidation ability in Halomonas sp. HAL1, while the complementary strain recovered the phenotype back to the wild type strain (Figure 1B).

The growth curves and As(III) oxidation efficiencies of the Halomonas sp. strains were tested in both low $\mathrm{Pi}(0.1 \mathrm{mM})$ and normal Pi (1 mM) conditions (Figures 2, 3). Consistent with the qualitative $\mathrm{KMnO}_{4}$ tests, the As(III) oxidation phenotypes disappeared at the low $\mathrm{Pi}$ condition in the phoR or $p h o B$ disruption strains, while the complemented strain HAL1- $\Delta p h o B$ $C$ reversed the mutants null phenotype back to wild type status (Figure 2). However, in the normal Pi condition, all of the four Halomonas sp. strains showed similar As(III) oxidation rates during the 7-day test (Figure 3), indicating that PhoBR may only have an effect on As(III) oxidation at the low Pi condition. The results are consistent with the properties of PhoBR because they are the regulators responsible for Pi stress (Hsieh and Wanner, 2010).

\section{PhoBR Influenced the Expression of aioBA}

To understand how $p h o B R$ and aioBA respond to As(III) in Halomonas sp. HAL1, quantitative lacZ reporter gene analyses were performed with the lac $Z$ reporter vector pCMlac $Z$ constructed in this study (Figure S2). The expressions of phoBR::lacZ and aioBA::lacZ were not induced (Figure 4A) in $5-$ or 6 -h cultivations in the low $\mathrm{Pi}$ condition without As(III). However, in the low Pi condition with the addition of $1 \mathrm{mM}$ As(III), the expressions of phoBR::lacZ and aioBA::lacZ increased with increasing induction time, and the phoBR::lacZ expression appeared to be higher than that of aioBA::lacZ (Figure 4A). Meanwhile, in the low Pi condition, the expression of aioBA::lac $Z$ was significantly induced by As(III) after $6 \mathrm{~h}$ of cultivation in strains HAL1 and HAL1- $\triangle p h o B-C$, but no statistically significant inductions in the mutants HAL1-phoR 931 and HAL1- $\triangle p h o B$ were observed (Figure 5A). However, in the normal Pi condition, the expression levels of phoBR::lacZ were similar in the presence or absence of As(III), and the expression of aioBA::lacZ was again significantly induced by As(III) (Figure 4B). In addition, the disruption of $p h o B$ and $p h o R$ had no effect on the expression of aioBA::lac $Z$ in the normal Pi condition (Figure 5B). The expression of aioBA in low $\mathrm{Pi}$ and normal $\mathrm{Pi}$ conditions were consistent with the As(III) oxidation phenotypes (Figures 2, 3). The above results indicated that $\mathrm{PhoBR}$ regulates the expression of aioBA in low $\mathrm{Pi}$ condition but not in normal $\mathrm{Pi}$ condition.

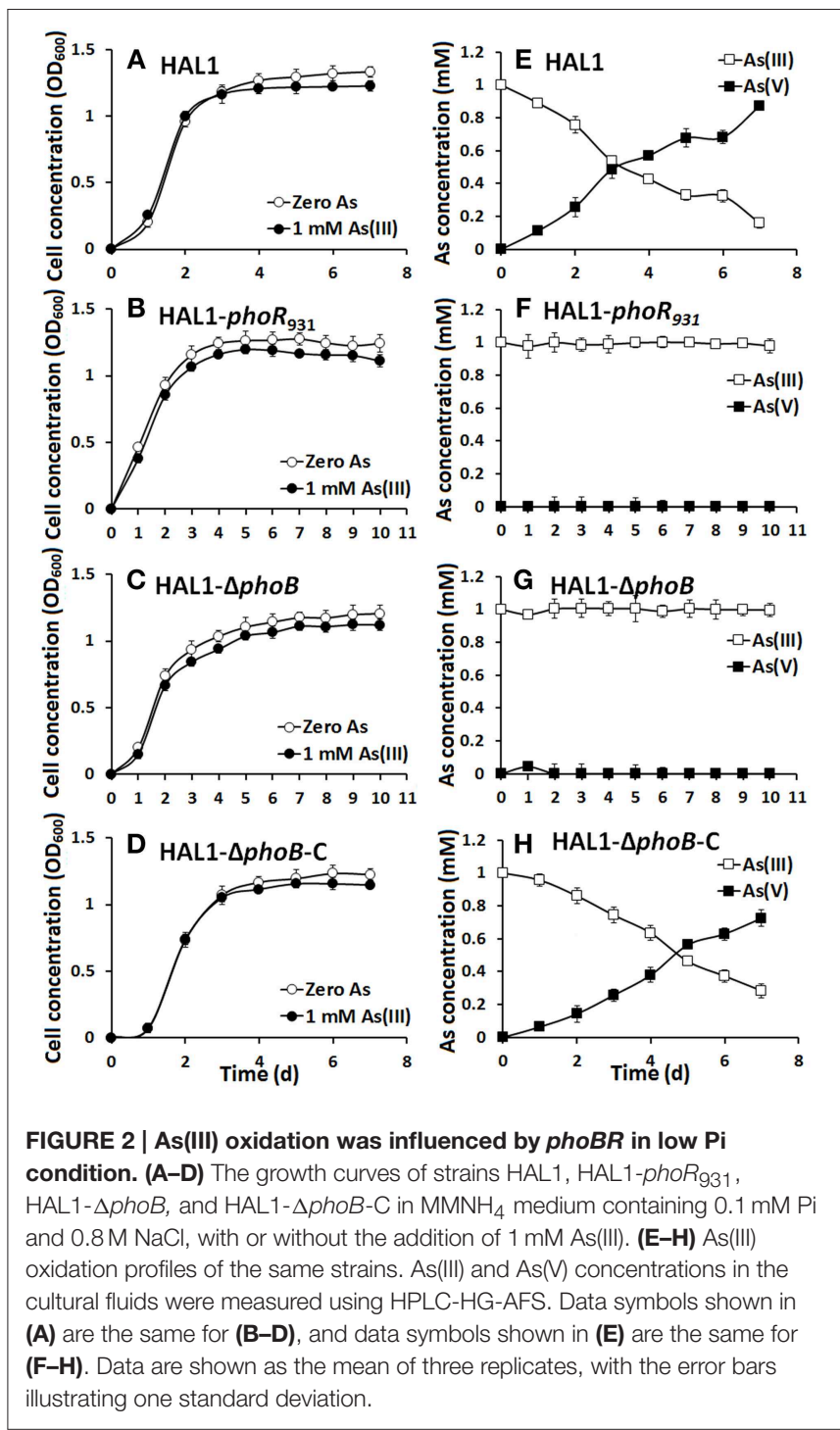

\section{PhoB Binds to the Regulatory Region of aioBA}

Based on 15 reported Pho box sequences (Yuan et al., 2006), we predicted a Pho motif using the MEME on-line program (http:// meme.nbcr.net/meme/cgi-bin/meme.cgi; Bailey and Elkan, 1994; Figure 6A). With the predicted Pho motif, we found a putative Pho box (TTGACACTCCATTGTTAT) located in the antisense strand within -21 to -3 bp upstream from the aioB start codon (Figure 6B); this box is $56 \%$ identical to the E. coli consensus Pho box (Diniz et al., 2011).

To examine the interaction between $\mathrm{PhoB}$ and the putative Pho box, we first used a bacterial one-hybrid system to test the protein-DNA interaction based on the transcriptional activation of HIS3 and aadA (Guo et al., 2009). The regulatory sequence of aioBA containing the putative Pho box was cloned into HIS3aadA upstream of the reporter vector $\mathrm{pBXcmT}$, while the PhoB coding region was introduced into $\mathrm{PTRG}$ vector. Both of the two constructed vectors were then transferred into a reporter strain. The reporter strain containing $\mathrm{pBX} \mathrm{cmT}$-Paio $B A$ and $\mathrm{pTRG}-\mathrm{pho} B$ 

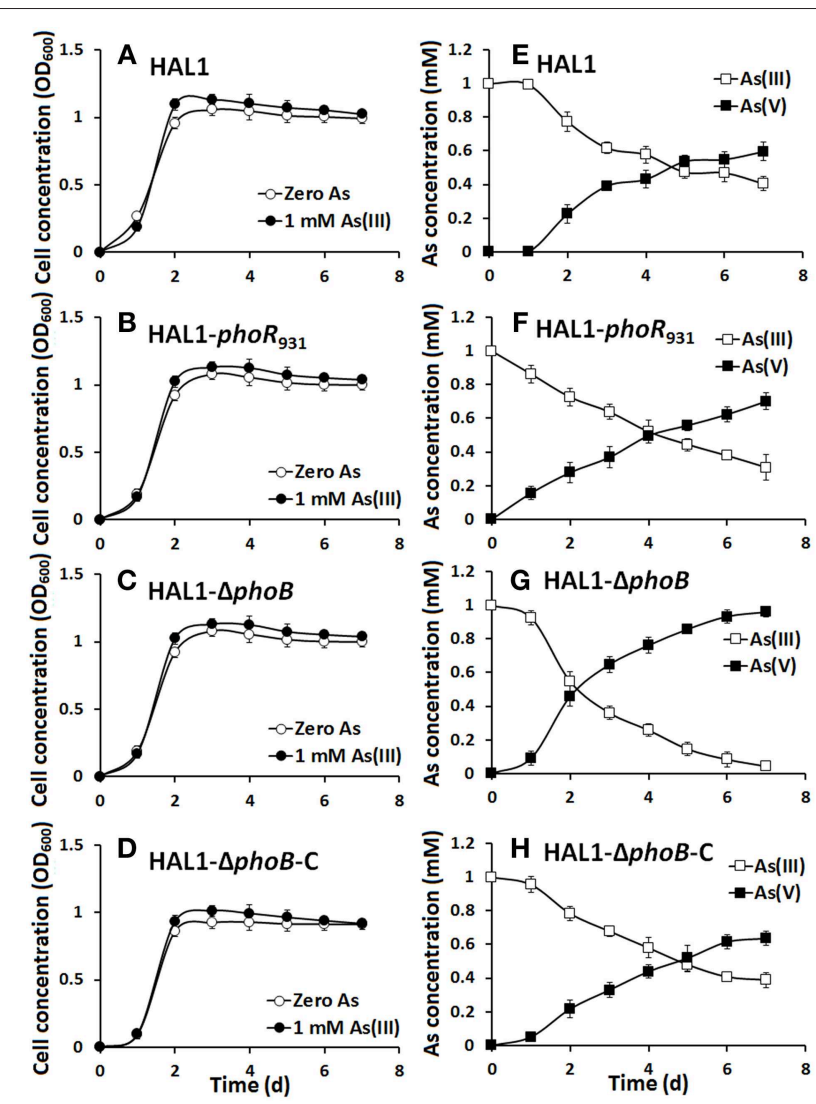

FIGURE 3 | As(III) oxidation was not influenced by phoBR in normal Pi condition. (A-D) The growth curves of strains HAL1, HAL1-phoR ${ }_{931}$, $\mathrm{HAL} 1-\Delta p h o B$, and HAL1- $\Delta$ phoB-C in $\mathrm{MMNH}_{4}$ medium with the presence of $1 \mathrm{mM} \mathrm{Pi}$ and $0.8 \mathrm{M} \mathrm{NaCl}$, with or without the addition of $1 \mathrm{mM} \mathrm{As}(\mathrm{III})$. (E-H) $\mathrm{As}(\mathrm{III})$ oxidation profiles of the same strains. As(III) and $\mathrm{As}(\mathrm{V})$ concentrations in the cultural fluids were measured using HPLC-HG-AFS. Data symbols shown in (A) are the same for (B-D), and data symbols shown in (E) are the same for (F-H). Data are shown as the mean of three replicates, with the error bars illustrating one standard deviation.

grew well on the screening plate containing 3-AT and Str, and the negative control (the reporter strain containing $\mathrm{pBX} \mathrm{cmT}$-PaioBA and pTRG) did not grow, which indicated that PhoB could interact with the aioBA regulatory region in vivo (Figure 7A).

A purified $\mathrm{His}_{6}$-tag $\mathrm{PhoB}$ (Figure S3) and a 209-bp aioBA regulatory sequence containing the putative Pho box were then used to test the interaction between $\mathrm{PhoB}$ and the putative Pho box in vitro using EMSA. With increasing PhoB concentration, the free DNA substrates gradually disappeared, while the intensity of the shifted DNA band increased (Figure 7B). Neither heat-denatured PhoB nor non-specific DNA (excluding the putative Pho box) exhibited a retardation band in the EMSA gel (Figure 7B). Moreover, the unlabeled DNA substrate could competitively inhibit the binding of $\mathrm{PhoB}$ to the labeled DNA substrate (Figure 7B). The results indicated that $\mathrm{PhoB}$ could bind specifically to the aio $B A$ regulatory region.

Based on the comparison of the Pho boxes between E. coli and the regulatory region of the aioBA genes, the conserved base pairs in the 18 bp Pho box were predicted (Figure 8A). To test

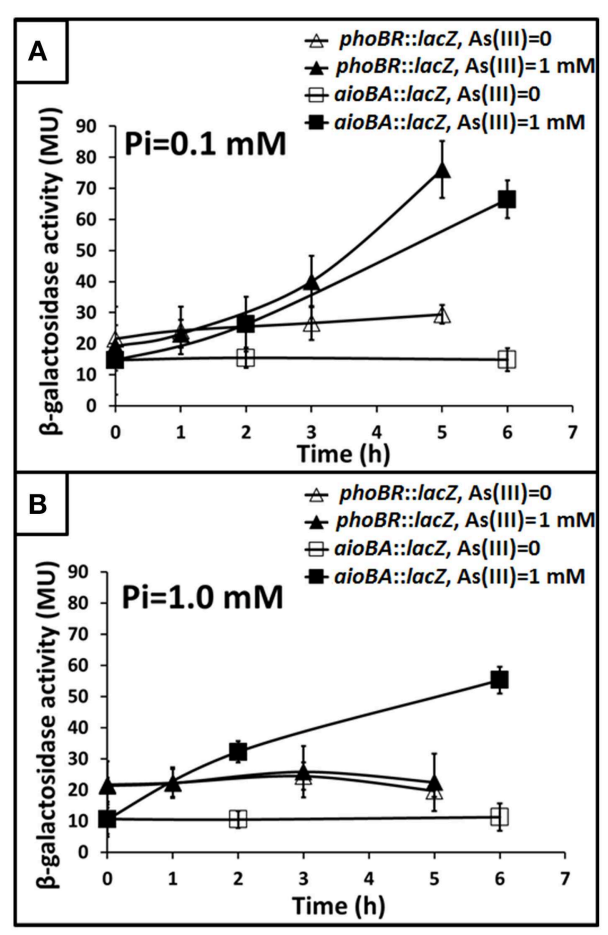

FIGURE 4 | Influence of As(III) on the expression of phoBR and aioBA. Expression of phoBR::lacZ and aioBA::lacZ in strain HAL1 were monitored. Data are shown as the mean of three replicates, with the error bars illustrating one standard deviation. (A) Bacteria were cultured in $\mathrm{MMNH}_{4}$ medium containing $0.1 \mathrm{mM} \mathrm{Pi}$ and $0.8 \mathrm{M} \mathrm{NaCl}$, with or without the addition of $1 \mathrm{mM}$ As(III). (B) Bacteria were cultured in $\mathrm{MMNH}_{4}$ medium containing $1 \mathrm{mM}$ Pi and $0.8 \mathrm{M} \mathrm{NaCl}$, with or without the addition of $1 \mathrm{mM} \mathrm{As}(\mathrm{III})$.

the exact binding sequence of PhoB in the regulatory region of aioBA genes, a short DNA fragment containing the putative Pho box (18 bp) and its bilateral five bases (protection bases) were used for EMSA testing (Box1, Figure 8B), and the conserved nine base pairs were site-directed substituted separately, generating Box2, Box3, and Box4 (Figure 8B). As shown in Figure 8C, PhoB was capable of binding with substrates containing the putative Pho box (Box1), but was incapable of binding with the mutated sequences Box2, Box3, and Box4. This indicated that the 18-bp putative Pho box (TTGACACTCCATTGTTAT) is essential for $\mathrm{PhoB}$ to bind with the aioBA regulatory region, and the nine conserved bases (Figure 8A) are the critical recognition sites.

\section{Discussion}

Currently, the regulation models of As(III) oxidation have only been reported in the As(III)-oxidizing strains that contain aioXSR in their genomes (Kang et al., 2012a,b; Liu et al., 2012; Li et al., 2013; Moinier et al., 2014). However, the potential proteins involved in the regulation of As(III) oxidase genes in As(III)-oxidizing strains without aioXSR, such as Halomonas sp. HAL1 (Lin et al., 2012), remain unknown. In this study, after discovering that the phoR mutant affected the As(III) oxidation phenotypes by transposon mutagenesis, we predicted that PhoBR may regulate the expression of As(III) oxidase genes in strain 


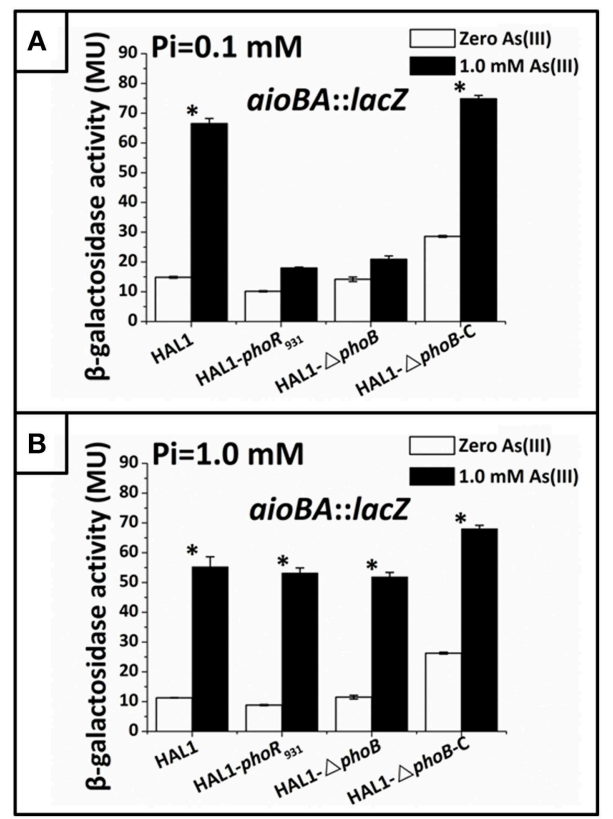

FIGURE 5 | Quantitative lacZ reporter gene analysis of aioBA::lacZ expression in strains HAL1, HAL1-phoR ${ }_{931}$, HAL1- $\Delta$ phoB, and HAL1- $\Delta$ phoB-C. $\beta$-galactosidase activity is presented as Miller units. Data are shown as the mean of three replicates, with the error bars represent \pm 1 $\mathrm{SD}$. (A) Bacteria were cultured in $\mathrm{MMNH}_{4}$ medium containing $0.1 \mathrm{mM} \mathrm{Pi}$ and $0.8 \mathrm{M} \mathrm{NaCl}$, with or without the addition of $1 \mathrm{mM} \mathrm{As}(\mathrm{III})$. With the addition of $\mathrm{As}(\mathrm{III})$, the mean values of strain HAL1 and HAL1- $\triangle$ phoB-C were significantly different from the ones with the absence of $A s(I I I) ~\left({ }^{*} p<0.05\right)$. (B) Bacteria were cultured in $\mathrm{MMNH}_{4}$ medium containing $1 \mathrm{mM} \mathrm{Pi}$ and $0.8 \mathrm{M} \mathrm{NaCl}$, with or without the addition of $1 \mathrm{mM} \mathrm{As}(\mathrm{III})$. With the addition of $\mathrm{As}(\mathrm{III})$, the mean values of all the four strains were significantly different from the ones with the absence of $\mathrm{As}(\mathrm{III})\left({ }^{*} p<0.05\right)$.

HAL1 because PhoBR can activate/inhibit the transcription of numerous genes (Yuan et al., 2006; Lamarche et al., 2008; Hsieh and Wanner, 2010).

Subsequently, we conducted a comprehensive analysis to assess this hypothesis. We concluded that PhoBR could regulate the expression of aioBA under low-Pi condition based on the following observations: (i) the mutants of $p h o R$ or $p h o B$ affected the As(III) resistance and oxidation phenotypes under low $\mathrm{Pi}$ condition; (ii) PhoBR influenced the expression of aioBA; (iii) As(III) induced the expression of PhoBR; and (iv) PhoB could bind with the putative pho box located in the regulation region of aioBA.

We also found that low Pi levels increased the As(III) oxidation efficiency in strain HAL1 and is consistent with the report showing that bacterial As(III) oxidation is more efficient in low Pi condition than in normal or high-Pi conditions (Kang et al., 2012b; Wang et al., 2015). In A. tumefaciens that containing AioXSR, the Pi stress response genes ( $p h o B, p s t S$, and $p h o U$ ) are intricately co-regulated with the As(III) oxidation genes aioBA, and the regulatory cross-talk via phosphorelay to PhoB from AioS has been proposed (Kang et al., 2012b), in which AioXSR plays the dominant role in As(III) oxidation. Although the Pi stress response proteins are related to As(III) oxidation, the

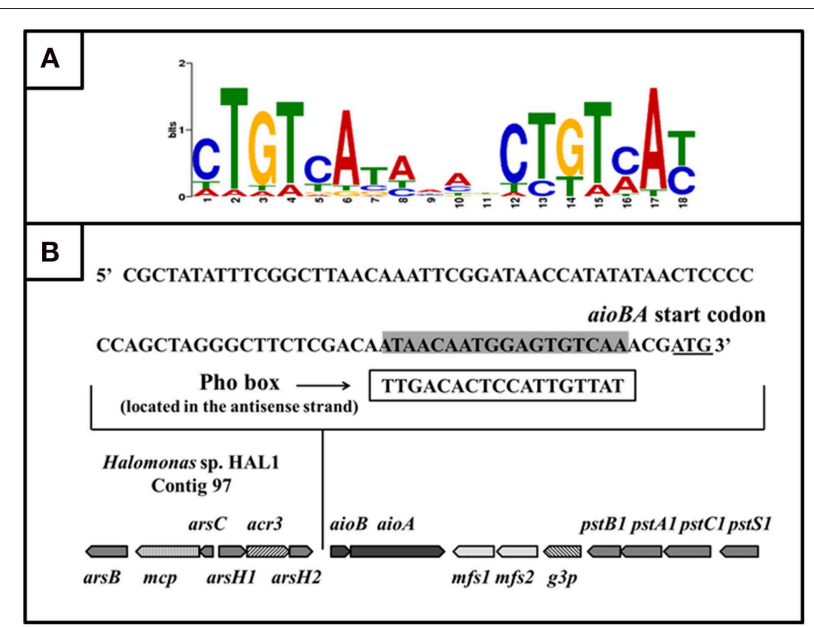

FIGURE 6 | The putative PhoB binding site (Pho box) in the aioBA promoter region. (A) Sequence logo of Pho motif predicted by MEME program based on the known Pho boxes. Higher bit scores indicate more conservation at respective site. (B) The putative Pho box sequence was denoted by rectangular box, which is reverse complement with the sequence in sense strand (Labeled gray).

regulatory relationship between $\mathrm{PhoBR}$ and As(III) oxidation appeared to be indirect in strain $5 \mathrm{~A}$, since no recognizable Pho box was found in the aioSRBA regulation region (Kang et al., 2012b). In this study, the aioXSR are absent in strain HAL1, but the low $\mathrm{Pi}$ concentration still showed effect on As(III) oxidation and the expression of $p h o B$ was also induced by As(III), similar to A. tumefaciens 5A (Kang et al., 2012b). These results imply that As and $\mathrm{Pi}$ metabolisms are also likely to be co-regulated in strain HAL1. However, the co-regulation mechanisms should be different between the strains HAL1 and $5 \mathrm{~A}$, since the suggested cross-talk phosphorylation between the AioXSR and PhoRB (Kang et al., 2012b) cannot be exist in strain HAL1. In addition, since strain HAL1 does not carry aioXSR, the co-regulation between $\mathrm{As}$ and $\mathrm{Pi}$ are more likely related to the concentrations As and Pi which needs to be further investigated.

Even though the PhoBR was reported in numerous studies to respond to Pi stress, the PhoB or PhoR have never showed to bind Pi directly, instead, PstS was the protein to bind $\mathrm{Pi}, \mathrm{As}(\mathrm{V})$ (Hsieh and Wanner, 2010; Wang et al., 2015), and possibly As(III). In some situations, the two-component system does not response environmental signals directly. For example, in our previous study with $A$. tuemfaciens $5 \mathrm{~A}$, even though the As(III)-response two-component system AioSR regulates the expression of As(III) oxidase genes aioBA, there is no As(III) binding domain in AioS or AioR. Instead, a protein called AioX binds As(III) directly (Liu et al., 2012), and AioX possibly transfers the As(III) signal to AioS. In our study, it is also possible that the "PstS" has the ability to sense Pi or As(III) and transfer the signals to PhoR in strain HAL1. PhoR auto-phosphorylates and transfers Pi group to PhoB, which then binds to the aioBA promoter region containing a putative Pho box and transcriptionally activates the expression of aioBA in an As(III)-dependent manner. 


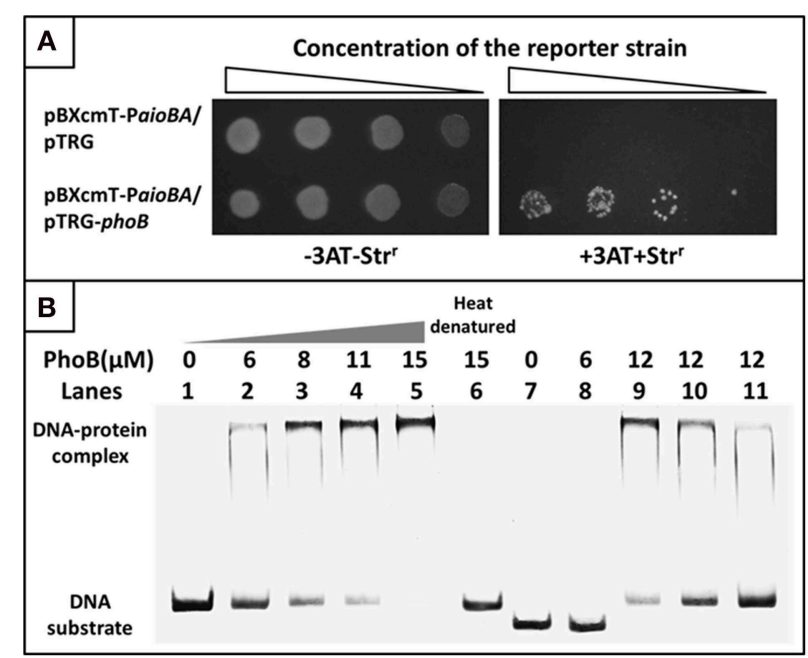

FIGURE 7 | Bacterial one-hybrid system and EMSA analyses for the interaction between PhoB and the aioBA promoter region. (A) Bacterial one-hybrid assay. A co-transformant containing vector PTRG and pBXcmT-PaioBA was used as the self-activation control. -3AT-Str represents the LB plate, +3AT+Str represents the screening plate. (B) EMSA assays. Lanes 1-6, 1.6 pmol FAM-labeled aioBA regulatory region DNA were co-incubated with various amounts of PhoB activated or heat-denatured; Lanes 7-8, 1.6 pmol FAM-labeled DNA sequence without putative Pho box were co-incubated with PhoB; Lanes 9-11, the competition assay using 1.6 pmol FAM-labeled aioBA regulatory region DNA and $12 \mu \mathrm{M}$ PhoB against 1.4, 2.7, and $6.1 \mathrm{pmol}$ unlabeled aioBA regulatory region DNA.

Based on the results of this study, the regulation of As(III) oxidation in different As(III)-oxidizing strains can be classified into three models: (i) the regulation model of the three-component system, AioXSR (Liu et al., 2012); (ii) the co-regulation model of AioXSR with the metalloprotein AioF belonging to the ArsR/SmtB family (Moinier et al., 2014); and (iii) the regulation model manipulated by a two-component system, PhoBR (this study). Since the As(III)-oxidizing strains that lack aioXSR genes were reported increasingly (Cai et al., 2009; Osborne et al., 2013; Koechler et al., 2015), the PhoBR regulation model may be widespread in these As(III)-oxidizing strains. Pi is generally present at low concentrations in terrestrial ecosystems (Vieira et al., 2008), thus the regulation of aioBA expression by PhoBR is reasonable when AioXSR is absent in As(III)-oxidizing strains. Moreover, it is obvious that As(III) oxidation was unaffected by the mutation of $p h o B$ or $p h o R$ under normal Pi conditions. Since Pit system was reported to involve in the Pi acquisition under normal Pi conditions (Hsieh and Wanner, 2010), it is very likely that the regulation models are different between low and normal Pi conditions.

\section{Materials and Methods}

\section{Bacterial Strains and Growth Conditions}

The bacterial strains and plasmids used for this study are listed in Table S1, while the primers are listed in Table S2. Halomonas sp. HAL1 and its derivative strains were cultured at $28^{\circ} \mathrm{C}$ in a defined minimal mannitol medium $\left(\mathrm{MMNH}_{4}\right)$
(Somerville and Kahn, 1983) containing $0.1 \mathrm{mM}$ or $1 \mathrm{mM}$ $\mathrm{Pi}\left(\mathrm{K}_{2} \mathrm{HPO}_{4} \cdot 3 \mathrm{H}_{2} \mathrm{O}: \mathrm{KH}_{2} \mathrm{PO}_{4}=4: 1, \mathrm{pH}=7.2\right)$ and $0.8 \mathrm{M}$ $\mathrm{NaCl}$. When needed, $1 \mathrm{mM} \mathrm{NaAsO}{ }_{2}$ [As(III)] was added into the medium. E. coli strains were grown at $37^{\circ} \mathrm{C}$ in LuriaBertani (LB) medium. Stock solutions of kanamycin (Kan, 50 $\mathrm{mg} / \mathrm{mL}$ ), chloramphenicol $(\mathrm{Cm}, 25 \mathrm{mg} / \mathrm{mL})$, tetracycline (Tet, $5 \mathrm{mg} / \mathrm{mL})$, and streptomycin $(\mathrm{Str}, 8 \mathrm{mg} / \mathrm{mL})$ were added when required.

\section{Transposon Mutagenesis in Combination with Qualitative $\mathrm{KMnO}_{4}$ Screening to Isolate Arsenite Oxidation Mutant}

A Kan resistance transposon delivery vector, pRL27, encoding a hyperactive Tn5 transposase was used to construct a transposon mutant library in this study (Larsen et al., 2002). E. coli strain S17-1 was employed to transfer pRL27 into the recipient strain Halomonas sp. HAL1. The transformants were selected on $\mathrm{MMNH}_{4}$ agar plates (Somerville and Kahn, 1983) containing $0.1 \mathrm{mM} \mathrm{Pi}$ and $0.8 \mathrm{M} \mathrm{NaCl}$ with $50 \mu \mathrm{g} / \mathrm{mL}$ Kan. After $4 \mathrm{~d}$ cultivation at $28^{\circ} \mathrm{C}$, the colonies were inoculated into the same liquid medium in a 96-well plate containing $0.1 \mathrm{mM} \mathrm{Pi}$, $0.8 \mathrm{M} \mathrm{NaCl}, 50 \mu \mathrm{g} / \mathrm{mL} \mathrm{Kan}$, and $200 \mu \mathrm{M}$ As(III). Followed by $7 \mathrm{~d}$ cultivation at $28^{\circ} \mathrm{C}$ with shaking at $100 \mathrm{rpm}$, qualitative $\mathrm{KMnO}_{4}$ screening was used to detect the As(III) oxidation phenotypes of transformants (Fan et al., 2008). Using the As(III)oxidizing strain HAL1 as a positive control, the candidate mutant strains were screened based on the yellow color tested with $\mathrm{KMnO}_{4}$, which indicates the disability of As(III) oxidation. Then, DNA of each potential mutant was extracted, digested with BamHI (Fermentas) and self-ligated using T4 DNA ligase (Promega) before being transferred into E. coli strain S17-1. The transformants were selected on LB agar plates containing $50 \mu \mathrm{g} / \mathrm{mL} \mathrm{Kan}$, and then after $12-24 \mathrm{~h}$ of cultivation at $37^{\circ} \mathrm{C}$, the plasmid of each transformant was extracted. The primers pRLSR and pRLSF (Table S2) were used to amplify the flanking sequences of Tn 5 transposon by inverse PCR. After the sequencing, the insertion sites were analyzed using the NCBI BLAST server (http://blast.ncbi.nlm.nih.gov/Blast.cgi) based on the genome of strain HAL1 (Lin et al., 2012).

\section{Construction of phoB Mutant and the Complemented Strains}

The suicide allelic exchange vector pCM184 was used to construct the phoB mutant in Halomonas sp. HAL1 (Marx and Lidstrom, 2002). The bilateral flanking regions of phoB were amplified by PCR using the primers phoB-up-F/phoB-up$\mathrm{R}$ and phoB-down-F/phoB-down-R (Table S2). The upstream PCR fragment was then cloned into AatII-BsrGI sites, while the downstream PCR fragment was introduced into the SacIISacI sites of pCM184. The resulting phoB allelic exchange vector pCM184-BUD was then mobilized into strain HAL1 via conjugation with $E$. coli strain S17-1, and the double crossing over phoB mutants were selected using $50 \mu \mathrm{g} / \mathrm{mL}$ Kan. Followed by screening with $25 \mu \mathrm{g} / \mathrm{mL}$ Tet, the Tet-sensitive and Kan-resistant mutants were identified by PCR using the primers phoBupYZ-F/phoB-upYZ-R and phoB-downYZ-F/phoB-downYZ-R (Table S2). 


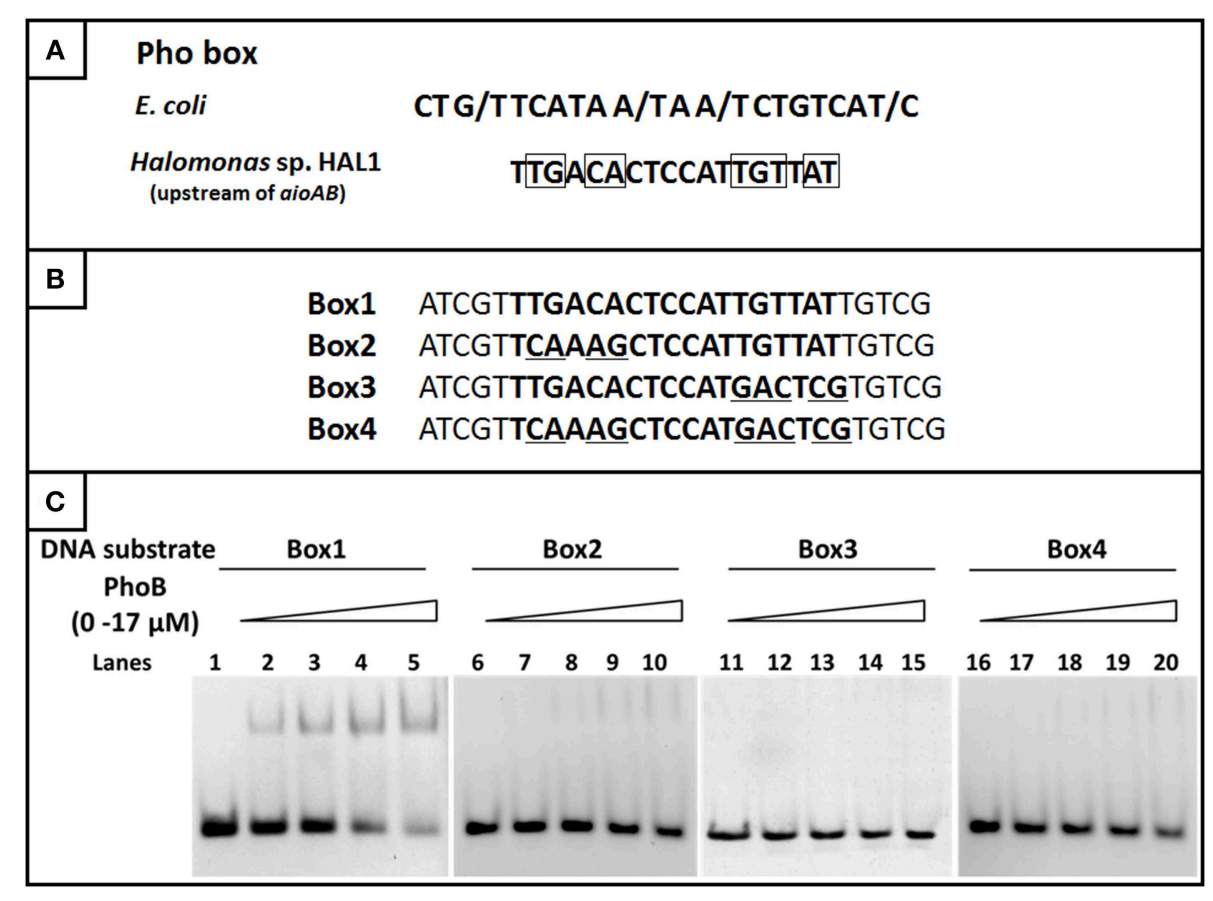

FIGURE 8 | EMSA for identifying the binding sites in the aioBA promoter region. (A) The comparison of the consensus Pho box in E. coli and the putative Pho box in the aioBA regulatory region. The optional bases were marked by slash, while the conserved bases in the Pho box of aioBA were marked with rectangle. (B) The four tested DNA substrates. All of the tested binding sequences were shown in bold. Box1 is the original Pho box in the aioBA regulatory region. Box2, Box3, and Box4 were mutated in different conserved bases. The mutation base pairs were marked with underline. (C) EMSA for the DNA-binding activity of PhoB with different substrates. Each of four oligonucleotide substrates was mixed with 0-17 $\mu \mathrm{M}$ PhoB. PhoB could bind with Box1, but not with Box2, Box3, or Box4 which were mutated in the conserved sites.

A broad host vector pCT-Zori was constructed (see the Supplementary Materials) and used to generate the complementary strain of $p h o B$ mutant. For $\triangle p h o B$ complementation, the complete phoBR coding region along with the 450-bp upstream regulatory region was PCR amplified and cloned into the SacI and HindIII sites of pCT-zori, resulting in pCT-zori-phoBR. This plasmid was transformed into E. coli strain S17-1 and conjugated with strain HAL1- $\triangle$ phoB. The complementary strain HAL1- $\triangle p h o B-\mathrm{C}$ was selected by $\mathrm{Cm}$ resistance and confirmed by PCR using primers PhoB-HB-F/ PhoB-HB-R (Table S2) along with sequencing.

\section{Growth and As(III) Oxidation Tests}

The cultures of HAL1, HAL1-phoR ${ }_{931}$, HAL1- $\triangle p h o B$, and HAL1- $\triangle$ phoB-C $\left(\mathrm{OD}_{600} \sim 0.5\right)$ were each inoculated $(1 \mathrm{~mL})$ into $100 \mathrm{~mL} \mathrm{MMNH}_{4}$ containing $0.1 \mathrm{mM}$ or $1 \mathrm{mM} \mathrm{Pi}$ and $0.8 \mathrm{M} \mathrm{NaCl}$ in the presence or absence of $1 \mathrm{mM} \mathrm{As}$ (III). They were then cultured at $28^{\circ} \mathrm{C}$ by shaking at $160 \mathrm{rpm}$ for up to $10 \mathrm{~d}$. Each day, culture samples were taken for monitoring optical density $\left(\mathrm{OD}_{600}\right)$ using a spectrophotometer (Beckman DU800; Beckman, Fullerton, CA) and quantifying $\mathrm{As}(\mathrm{III})$ and $\mathrm{As}(\mathrm{V})$ with a combination of high-performance liquid chromatography with hydride-generation atomic fluorescence spectroscopy (HPLC-HG-AFS) (Beijing Titan Instruments Co., Ltd., China), as previously described (Liao et al., 2013).

\section{Reporter Gene Assays of aioBA and phoBR}

The reporter gene assays in this study was tested by $\beta$ galactosidase activity (Miller, 1972). The promoter regions of aioBA and $p h o B R$ were amplified from strain HAL1 (primers listed in Table S2) and introduced into the BglII-BsrGI sites of pCM-lacZ (see the Supplementary Materials), respectively. The resulting plasmids were then introduced into Halomonas sp. strains via biparental conjugation. When the cell $\mathrm{OD}_{600}$ reached approximately 0.4 , the cells were harvested by centrifugation $(8000 \mathrm{rpm}, 10 \mathrm{~min})$ and inoculated with the same $\mathrm{OD}_{600}$ into the $\mathrm{MMNH}_{4}$ medium containing $0.8 \mathrm{M} \mathrm{NaCl}$ and $1 \mathrm{mM}$ As(III) along with the addition of $0.1 \mathrm{mM}$ or $1 \mathrm{mM}$ Pi. The cultures without As(III) were used as the negative controls. All of the cultures were cultured at $28^{\circ} \mathrm{C}$ on a rotary shaker. During the incubation, $\beta$-galactosidase assays were conducted as described previously (Miller, 1972; Kang et al., 2012b).

\section{Bacterial One-hybrid System Assay}

The Pho box-PhoB interaction was tested in vivo using a bacterial one-hybrid system as described previously (Guo et al., 2009). The phoB coding region was amplified (primers listed in Table S2) and cloned into the BamHI-EcoRI sites of the pTRG vector (Stratagene), generating $\mathrm{pTRG}-\mathrm{phoB}$. The regulatory region of aioBA including the putative Pho box was amplified (primers listed in Table S2) and inserted directly into the XcmI site of pBXcmT (Guo et al., 2009), yielding pBXcmT-PaioBA. Both of 
the two recombinant plasmids were co-transformed into the reporter strain E. coli XL1-Blue MRF' Kan (Stratagene). After 3$4 \mathrm{~d}$ of cultivation at $28^{\circ} \mathrm{C}$ on a selective screening medium plate containing $20 \mathrm{mM} 3$-amino-1,2,4-triazole (3-AT), $16 \mu \mathrm{g} / \mathrm{mL} \mathrm{Str}$, $15 \mu \mathrm{g} / \mathrm{mL}$ Tet, $34 \mu \mathrm{g} / \mathrm{mL} \mathrm{Cm}$, and $50 \mu \mathrm{g} / \mathrm{mL} \mathrm{Kan,} \mathrm{the} \mathrm{co-}$ transformant growth was tested (Guo et al., 2009). In addition, a co-transformant containing vector $\mathrm{pTRG}$ and $\mathrm{pBX} \mathrm{cmT}$-PaioBA was used as the self-activation control.

\section{Electrophoretic Mobility Shift Assay (EMSA)}

To identify the putative Pho box in the aioBA regulatory region, a 209-bp fragment containing the putative Pho box of the aioBA regulatory region was amplified using PaioA-F/PaioA-R (Table S2). The primer PaioA-F was labeled with fluorophore FAM when needed. EMSA was carried out with a 1.6-pmol labeled probe and increasing concentrations of $\mathrm{PhoB}$ (from 0 to $15 \mu \mathrm{M}$ ), while the heat-denatured $\mathrm{PhoB}$ and unspecific DNA (exclude putative Pho box) were used as negative controls. For competition assay, 1.4-, 2.7-, and 6.1-pmol unlabeled probes were added to reaction mixtures containing $12 \mu \mathrm{M}$ PhoB and the 1.6pmol labeled probe. All reaction mixtures were incubated at $4^{\circ} \mathrm{C}$ for $30 \mathrm{~min}$ in binding buffer [ $20 \mathrm{mM}$ Tris- $\mathrm{HCl}, \mathrm{pH} 7.0 ; 50 \mathrm{mM}$ $\mathrm{NaCl} ; 1 \mathrm{mM}$ dithiothreitol (DTT); $10 \mathrm{mM} \mathrm{MgCl}_{2} ; 100 \mu \mathrm{g} / \mathrm{ml}$ bovine serum albumin (BSA)] and then loaded onto a $4 \%$ native PAGE. Followed by $2 \mathrm{~h}$ of running at $100 \mathrm{~V}$ in $0.5 \times \mathrm{TBE}$ buffer, gels were exposed to a phosphor imaging system (Fujifilm FLA-5100).

To test the exact binding sequence of $\mathrm{PhoB}$ in the regulatory region of aioBA genes, a 28-bp DNA fragments

\section{References}

Bailey, T. L., and Elkan, C. (1994). Fitting a mixture model by expectation maximization to discover motifs in biopolymers. Proc. Int. Conf. Intell. Syst. Mol. Biol. 2, 28-36.

Blanco, A. G., Canals, A., Bernués, J., Solá, M., and Coll, M. (2011). The structure of a transcription activation subcomplex reveals how sigma(70) is recruited to PhoB promoters. EMBO J. 30, 3776-3785. doi: 10.1038/emboj.2011.271

Blanco, A. G., Canals, A., and Coll, M. (2012). PhoB transcriptional activator binds hierarchically to pho box promoters. Biol. Chem. 393, 1165-1171. doi: 10.1515/hsz-2012-0230

Cai, L., Rensing, C., Li, X., and Wang, G. (2009). Novel gene clusters involved in arsenite oxidation and resistance in two arsenite oxidizers: Achromobacter sp. SY8 and Pseudomonas sp. TS44. Appl. Microbiol. Biotechnol. 83, 715-725. doi: 10.1007/s00253-009-1929-4

Crépin, S., Chekabab, S. M., Le Bihan, G., Bertrand, N., Dozois, C. M., and Harel, J. (2011). The Pho regulon and the pathogenesis of Escherichia coli. Vet. Microbiol. 153, 82-88. doi: 10.1016/j.vetmic.2011.05.043

Diniz, M. M. P., Goulart, C. L., Barbosa, L. C., Farache, J., Lery, L. M. S., Pacheco, A. B. F., et al. (2011). Fine-tuning control of phoBR expression in Vibrio cholerae by binding of PhoB to multiple Pho boxes. J. Bacteriol. 193, 6929-6938. doi: 10.1128/JB.06015-11

Fan, H., Su, C., Wang, Y., Yao, J., Zhao, K., Wang, Y., et al. (2008). Sedimentary arsenite-oxidizing and arsenate-reducing bacteria associated with high arsenic groundwater from Shanyin, Northwestern China. J. Appl. Microbiol. 105, 529-539. doi: 10.1111/j.1365-2672.2008.03790.x

Guo, M., Feng, H., Zhang, J., Wang, W., Wang, Y., Li, Y., et al. (2009). Dissecting transcription regulatory pathways through a new bacterial onehybrid reporter system. Genome Res. 19, 1301-1308. doi: 10.1101/gr.0865 95.108 containing an 18 bp putative Pho box and bilateral 5bp protection bases, were synthesized by Tsingke (Tsingke Biological Technology Company, Beijing, China) and directly annealed in vitro. EMSA for the DNA binding activity of PhoB was carried out on different substrates using 14.5pmol probes with increasing concentrations of PhoB (0$17 \mu \mathrm{M})$.

\section{Author Contributions}

FC designed and performed the experiments and wrote the manuscript. YC, SW, YL, and XL participated in the experiments. QW wrote and revised the draft of the manuscript. GW designed the study and revised the draft of the manuscript. All authors read and approved the final manuscript.

\section{Acknowledgments}

The present study was supported by the National Natural Science Foundation of China (31170106). We would like to express our gratitude to Dr. Timothy R. McDermott (Montana State University) for the scientific comments.

\section{Supplementary Material}

The Supplementary Material for this article can be found online at: http://journal.frontiersin.org/article/10.3389/fmicb. 2015.00923

Hsieh, Y.-J., and Wanner, B. L. (2010). Global regulation by the sevencomponent Pi signaling system. Curr. Opin. Microbiol. 13, 198-203. doi: 10.1016/j.mib.2010.01.014

Kang, Y. S., Bothner, B., Rensing, C., and McDermott, T. R. (2012a). Involvement of RpoN in regulating bacterial arsenite oxidation. Appl. Environ. Microbiol. 78, 5638-5645. doi: 10.1128/AEM.00238-12

Kang, Y. S., Heinemann, J., Bothner, B., Rensing, C., and McDermott, T. R. (2012b). Integrated co-regulation of bacterial arsenic and phosphorus metabolisms. Environ. Microbiol. 14, 3097-3109. doi: 10.1111/j.1462-2920.2012.02881.x

Kashyap, D. R., Botero, L. M., Franck, W. L., Hassett, D. J., and McDermott, T. R. (2006). Complex regulation of arsenite oxidation in Agrobacterium tumefaciens. J. Bacteriol. 188, 1081-1088. doi: 10.1128/JB.188.3.1081-1088.2006

Koechler, S., Arséne-Ploetze, F., Brochier-Armanet, C., Goulhen-Chollet, F., Heinrich-Salmeron, A., Jost, B., et al. (2015). Constitutive arsenite oxidase expression detected in arsenic-hypertolerant Pseudomonas xanthomarina S11. Res. Microbiol. 166, 205-214. doi: 10.1016/j.resmic.2015.02.010

Kulp, T. R., Hoeft, S. E., Asao, M., Madigan, M. T., Hollibaugh, J. T., Fisher, J. C., et al. (2008). Arsenic(III) fuels anoxygenic photosynthesis in hot spring biofilms from Mono Lake, California. Science 321, 967-970. doi: 10.1126/science.1160799

Kulp, T. R., Hoeft, S. E., and Oremland, R. S. (2004). Redox transformations of arsenic oxyanions in periphyton communities. Appl. Environ. Microbiol. 70, 6428-6434. doi: 10.1128/AEM.70.11.6428-6434.2004

Lamarche, M. G., Wanner, B. L., Crépin, S., and Harel, J. (2008). The phosphate regulon and bacterial virulence: a regulatory network connecting phosphate homeostasis and pathogenesis. FEMS Microbiol. Rev. 32, 461-473. doi: 10.1111/j.1574-6976.2008.00101.x

Larsen, R. A., Wilson, M. M., Guss, A. M., and Metcalf, W. W. (2002). Genetic analysis of pigment biosynthesis in Xanthobacter autotrophicus Py2 using a new, highly efficient transposon mutagenesis system that is functional in a wide 
variety of bacteria. Arch. Microbiol. 178, 193-201. doi: 10.1007/s00203-0020442-2

Li, H., Li, M. S., Huang, Y., Rensing, C., and Wang, G. (2013). In silico analysis of bacterial arsenic islands reveals remarkable synteny and functional relatedness between arsenate and phosphate. Front. Microbiol. 4:10. doi: 10.3389/fmicb.2013.00347

Liao, S. J., Zhou, J. X., Wang, H., Chen, X., Wang, H. F., and Wang, G. J. (2013). Arsenite oxidation using biogenic manganese oxides produced by a deep-sea manganese-oxidizing bacterium, Marinobacter sp MnI7-9. Geomicrobiol. J. 30, 150-159. doi: 10.1080/01490451.2011.654379

Lin, Y., Fan, H., Hao, X., Johnstone, L., Hu, Y., Wei, G. H., et al. (2012). Draft genome sequence of Halomonas sp. Strain HAL1, a moderately halophilic arsenite-oxidizing bacterium isolated from gold-mine soil. J. Bacteriol. 194, 199-200. doi: 10.1128/JB.06359-11

Liu, G., Liu, M., Kim, E. H., Maaty, W. S., Bothner, B., Lei, B. F., et al. (2012). A periplasmic arsenite-binding protein involved in regulating arsenite oxidation. Environ. Microbiol. 14, 1624-1634. doi: 10.1111/j.1462-2920.2011.02672.x

Makino, K., Amemura, M., Kim, S. K., Nakata, A., and Shinagawa, H. (1993). Role of the sigma 70 subunit of RNA polymerase in transcriptional activation by activator protein PhoB in Escherichia coli. Genes Dev. 7, 149-160. doi: 10.1101/gad.7.1.149

Marx, C. J., and Lidstrom, M. E. (2002). Broad-host-range cre-lox system for antibiotic marker recycling in Gram-negative bacteria. Biotechniques 33, 1062-1067.

Miller, J. H. (1972). Experiments in Molecular Genetics, p 431. Cold Spring Harbor, NY: Cold Spring Harbor Laboratory Press.

Moinier, D., Slyemi, D., Byrne, D., Lignon, S., Lebrun, R., Talla, E., et al. (2014). An ArsR/SmtB family member is involved in the regulation by arsenic of the arsenite oxidase operon in Thiomonas arsenitoxydans. Appl. Environ. Microbiol. 80, 6413-6426. doi: 10.1128/AEM.01771-14

Osborne, T. H., Heath, M. D., Martin, A. C. R., Pankowski, J. A., HudsonEdwards, K. A., and Santini, J. M. (2013). Cold-adapted arsenite oxidase from a psychrotolerant Polaromonas species. Metallomics 5, 318-324. doi: 10.1039/C2MT20180A

Pratt, J. T., McDonough, E., and Camilli, A. (2009). PhoB regulates motility, biofilms, and Cyclic di-GMP in Vibrio cholerae. J. Bacteriol. 191, 6632-6642. doi: 10.1128/JB.00708-09

Rhine, E. D., Ní Chadhain, S. M., Zylstra, G. J., and Young, L. Y. (2007). The arsenite oxidase genes (aroAB) in novel chemoautotrophic arsenite oxidizers. Biochem. Biophys. Res. Commun. 354, 662-667. doi: 10.1016/j.bbrc.2007.01.004

Rosen, B. P. (1995). Resistance mechanisms to arsenicals and antimonials. J. Basic Clin. Physiol. Pharmacol. 6, 251-263. doi: 10.1515/JBCPP.1995.6.3-4.251

Santini, J. M., Sly, L. I., Schnagl, R. D., and Macy, J. M. (2000). A new chemolithoautotrophic arsenite-oxidizing bacterium isolated from a gold mine: phylogenetic, physiological, and preliminary biochemical studies. Appl. Environ. Microbiol. 66, 92-97. doi: 10.1128/AEM.66.1.92-97.2000
Sardiwal, S., Santini, J. M., Osborne, T. H., and Djordjevic, S. (2010). Characterization of a two-component signal transduction system that controls arsenite oxidation in the chemolithoautotroph NT-26. FEMS Microbiol. Lett. 313, 20-28. doi: 10.1111/j.1574-6968.2010.02121.x

Sharma, V. K., and Sohn, M. (2009). Aquatic arsenic: toxicity, speciation, transformations, and remediation. Environ. Int. 35, 743-759. doi: 10.1016/j.envint.2009.01.005

Silver, S., and Phung, L. T. (2005). Genes and enzymes involved in bacterial oxidation and reduction of inorganic arsenic. Appl. Environ. Microbiol. 71, 599-608. doi: 10.1128/AEM.71.2.599-608.2005

Somerville, J. E., and Kahn, M. L. (1983). Cloning of the glutamine synthetase I gene from Rhizobium meliloti. J. Bacteriol. 156, 168-176.

Srinivasan, V. B., Venkataramaiah, M., Mondal, A., Vaidyanathan, V., Govil, T., and Rajamohan, G. (2012). Functional characterization of a novel outer membrane porin KpnO, regulated by PhoBR two-component system in Klebsiella pneumoniae NTUH-K2044. PLoS ONE 7:18. doi: 10.1371/journal.pone.0041505

Szpirer, C. Y., Faelen, M., and Couturier, M. (2001). Mobilization function of the pBHR1 plasmid, a derivative of the broad-host-range plasmid pBBR1. J. Bacteriol. 183, 2101-2110. doi: 10.1128/JB.183.6.2101-2110.2001

Vieira, R. P., Gonzalez, A. M., Cardoso, A. M., Oliveira, D. N., Albano, R. M., Clementino, M. M., et al. (2008). Relationships between bacterial diversity and environmental variables in a tropical marine environment, Rio de Janeiro. Environ. Microbiol. 10, 189-199. doi: 10.1111/j.1462-2920.2007.01443.x

Wang, Q., Qin, D., Zhang, S., Wang, L., Li, J., Rensing, C., et al. (2015). Fate of arsenate following arsenite oxidation in Agrobacterium tumefaciens GW4. Environ. Microbiol. 17, 1926-1940. doi: 10.1111/1462-2920.12465

Wanner, B. L. (1996). "Phosphorous assimilation and control of the phosphate regulon," in Escherichia coli and Salmonella typhimurium Cellular and Molecular Biology, eds F.C. Neidhardt, R. I. Curtiss, J. L. Ingraham, E. C. Lin, K. B. J. Low, B. Magasanik, et al. (Washington, DC: American Society for Microbiology), 1357-1381.

Yuan, Z. C., Zaheer, R., Morton, R., and Finan, T. M. (2006). Genome prediction of PhoB regulated promoters in Sinorhizobium meliloti and twelve proteobacteria. Nucleic Acids Res. 34, 2686-2697. doi: 10.1093/nar/gkl365

Conflict of Interest Statement: The authors declare that the research was conducted in the absence of any commercial or financial relationships that could be construed as a potential conflict of interest.

Copyright (C) 2015 Chen, Cao, Wei, Li, Li, Wang and Wang. This is an open-access article distributed under the terms of the Creative Commons Attribution License (CC $B Y)$. The use, distribution or reproduction in other forums is permitted, provided the original author(s) or licensor are credited and that the original publication in this journal is cited, in accordance with accepted academic practice. No use, distribution or reproduction is permitted which does not comply with these terms. 